\section{PET-CT zur Therapiekontrolle beim NSCLC}

Nicht alle Patienten mit nicht kleinzelligem Bronchialkarzinom (NSCLC) sprechen auf Erlotinib an. Ob sich mit einer ${ }^{18}$ F-Fluordesoxyglukose-Positronenemissionstomografie (FDG PET-CT) das Ansprechen kontrollieren lässt?

\begin{abstract}
A ls die Studie begann, hatte sich die Stratifizierung der Patienten nach dem EGFR-Mutationsstadium noch nicht durchgesetzt. Die Selektion erfolgte daher nach klinischen Kriterien, die ein Ansprechen auf Erlotinib vermuten ließen. $\mathrm{Zu}$ Studienbeginn führten die Untersucher ein PET-CT und ein diagnostisches CT mit Kontrastmittel, nach sechs Wochen noch einmal ein PET-CT und nach zwölf
\end{abstract}

Wochen ein weiteres Kontrastmittel-CT durch. Primärer Endpunkt war, dass bei zwölf von 35 Patienten eine frühe Progression nach sechs Wochen auftrat. Außerdem wurden die radiologischen und die symptomatischen Ansprechraten nach sechs und zwölf Wochen verglichen.

Das Ansprechen nach sechs Wochen lag mit $32 \%$ höher als bei diesem Patientenkollektiv zu erwarten gewesen wäre,

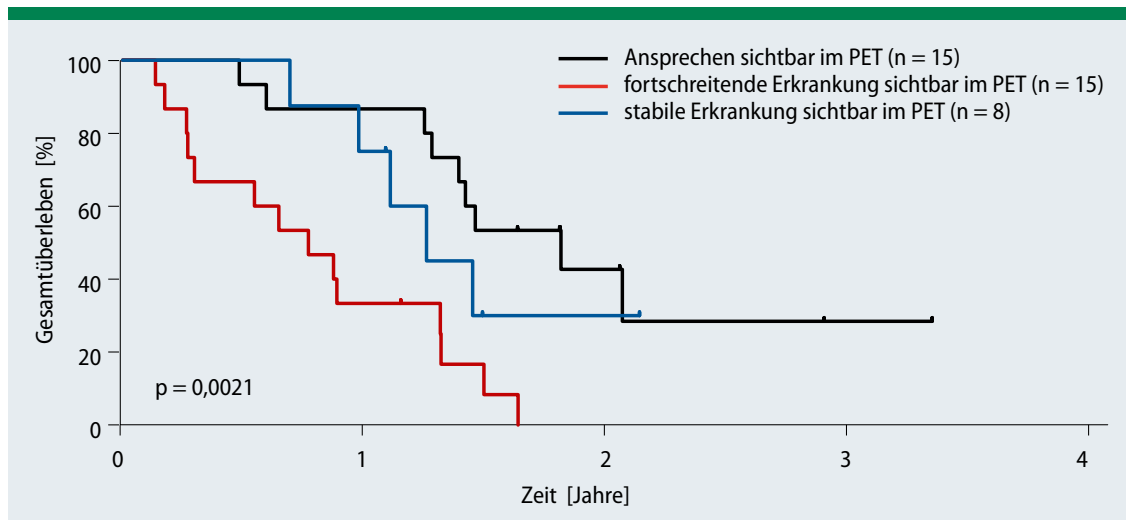

FDG PET-CT = 18-Fluordesoxyglukose Positronen-Emissions-Tomografie- Computertomografie

Abb.: Ein Ansprechen auf Erlotinib im FDG PET-CT korreliert mit dem Gesamtüberleben

\section{Im Stadium N2 bringt Bestrahlen keinen Vorteil}

Viele Patienten mit nicht kleinzelligem Bronchialkarzinom (NSCLC) im Stadium III erhalten nach R0-Resektion des Tumors noch eine Strahlentherapie mit dem Ziel, die Zahl lokaler Rezidive zu senken und die Überlebenschancen zu verbessern. Dieses Ziel wird aber laut einer aktuellen US-Studie nicht erreicht.

\footnotetext{
uan Wisnivesky von der Mount Sinai School of Medicine in New York und Kollegen analysierten retrospektiv Daten des SEER-Registers, das mit der Datenbank der Medicare-Krankenversicherung verlinkt werden kann und populationsbasiert und daher nicht von bestimmten Zuweisungsmustern abhängig ist. Identifiziert wurden 1.307 Patienten, die zwischen 1992 und 2005 an einem NSCLC mit Beteiligung der ipsilateralen mediastinalen oder subkarinalen Lymphknoten (N2) erkrankt waren. 710 davon (54\%) waren postoperativ bestrahlt worden. Ei-
}

nen Vorteil gegenüber nicht bestrahlten Patienten hatten sie davon nicht, die Überlebensraten waren nach einem und nach drei Jahren gleich $(\mathrm{p}=0,30)$. Daran änderte sich auch nichts, als man Faktoren in die Analyse einbezog, die sich vielleicht auf die Entscheidung für eine Radiotherapie ausgewirkt hatten (Komorbiditäten, Tumorgröße, postoperative Komplikationen).

Die Mortalität von Patienten nach Resektion eines Bronchialkarzinoms hängt stark vom Lymphknotenbefall ab. Sind die Knoten frei (pN0), leben rund $60 \%$ eventuell wegen der großen Zahl teilnehmender Frauen und Nichtraucher.

Es war eine gute Korrelation zwischen den partiellen Remissionstatus (PR) zu beiden Untersuchungszeitpunkten zu beobachten: Alle zehn Patienten mit PR nach sechs Wochen, waren auch noch nach zwölf Wochen progredient. Von 13 Patienten mit Progress nach zwölf Wochen hatten sieben diesen schon nach sechs Wochen gezeigt. Hier hätte man also die Therapie bereits nach sechs Wochen beenden können. Keiner der Patienten, die nach sechs Wochen eine stabile Erkrankung oder eine Progression aufwiesen, sprach später noch auf Erlotinib an. Das symptomatische Ansprechen korrelierte dabei nicht gut mit dem Ansprechen in der Bildgebung.

Fazit: Die Behandlung von mehr als zwölf Patienten (15 von 38) hätte man nach dem PET-CT nach sechs Wochen stoppen können. Daher scheint ein Beurteilen des Ansprechens per PET-CT zu diesem Zeitpunkt sinnvoll.

Friederike Klein

O'Brien MER et al. A phase Il study of ${ }^{18} \mathrm{~F}$-fluorodeoxyglucose $\mathrm{PET}-\mathrm{CT}$ in non-small cell lung cancer patients receiving erlotinib (Tarceva); objective and symptomatic responses at 6 and 12 weeks.

Eur J Cancer. 2012;48(1): 68-74.

der Betroffenen auch fünf Jahre nach der Diagnose noch; bei einem pN2-Befund sinkt die Quote auf $22 \%$. Der Versuch, die Überlebenschancen von Patienten mit N2-Tumoren durch Bestrahlung zu verbessern, ist daher verständlich, offenbar aber vergeblich.

Fazit: „Dieses Ergebnis sollte Konsequenzen für die Klinik haben“, fordern die Autoren. Denn die SEER-Daten zeigten, dass trotz fehlender Wirkungsnachweise ein großer Prozentsatz dieser Patienten eine Strahlentherapie erhalte. Randomisierte und kontrollierte Studien sollen den Effekt der Radiatio nun genauer bewerten helfen.

Robert Bublak

Wisnivesky JP et al. Postoperative radiotherapy for elderly patients with stage III lung cancer. Cancer. 2012; DOI: 10.1002/cncr.26585. 